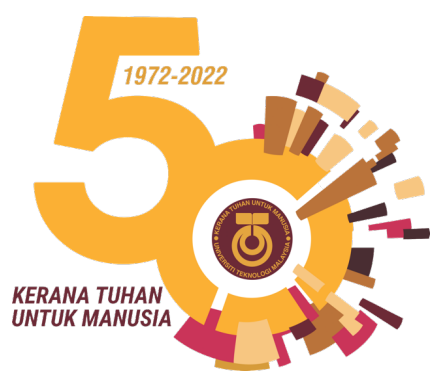

\title{
Dynamical System Analysis of the Prey- predator Interactions Involving Prey Refuge and Herd Behaviors in Preys
}

\author{
Zati Iwani Abdul Manafa,b,*, Mohd Hafiz Mohd ${ }^{\mathrm{b}}$ \\ a Faculty of Computer and Mathematical Sciences, Universiti Teknologi MARA \\ Kelantan, 18500 Machang, Kelantan, Malaysia; b School of Mathematical Science, \\ Universiti Sains Malaysia,11800 USM Penang, Malaysia.
}

Abstract By employing a prey refuge mechanism, more preys can be protected from predation. Prey species are also better protected from predation when they congregate in herds. However, what if the prey refuge and herd behavior mechanisms were combined in a system? To investigate this phenomenon, we consider two different prey-predator systems with prey refuge capacity. The first system is a simple prey-predator with prey refuge, whereas the second system considers prey refuge and prey herd behavior mechanisms. Using these models, we explore how different prey refuge strategies affect species interactions in both systems. To accomplish this, we use theoretical techniques (e.g., computing steady states and performing the stability analysis) and numerical bifurcation analysis to demonstrate various dynamical behaviors of these two prey-predator systems. Once prey refuge is treated as a bifurcation parameter, we observe the occurrence of supercritical Hopf and transcritical bifurcations in both systems. Furthermore, we explore the dynamic effects of prey refuge and predator handling time on species population interactions: our findings reveal that using both prey refuge and herd behavior as escape strategies; it is possible to dilute the predation pressure and ensure species biodiversity.

Keywords: Prey-predator, prey refuge, group defense, numerical bifurcation analysis, herd behaviour.

${ }^{*}$ For correspondence: zati431@uitm.edu.my

Received: 11 Sept 2021 Accepted: 14 Jan 2022

(C) Copyright Manaf and Mohd. This article is distributed under the terms of the Creative Commons Attribution License, which permits unrestricted use and redistribution provided that the original author and source are credited.

\section{Introduction}

In general, understanding the interactions between different species such as prey-predator interactions are a central goal in ecology. Prey refuge corresponds to how prey employs escape mechanism in their populations to seek protection from the predator's attack. The refuge process impacts population expansion because it reduces the probability of prey death by dispersing predator pressure [1]. Therefore, a prey refuge mechanism offers some degree of protection for preys against a predator.

Various researchers, including [1-6], have explored the dynamic effects of prey - predator systems in the involvement of refuge. Kar [2] introduced a prey-predator system with a response function of Holling type II that includes a constant proportion of prey refuges. They claim that expanding the refuge may lead to higher prey intensities and population expansions [2]. Apart from that, Chen and Wang [4] proposed an alternative prey-predator system that includes refuge capacity and herd behaviour mechanism in prey species. By utilizing prey refuges as a control measure, it is possible to reduce predation rates and effectively control predator density to prevent population disappearance and encourage coexistence.

Motivated by these ecological observations, we are particularly interested in the combined influence of refuge process and herd behaviour mechanism inspired by the ecological systems of Kar [2] and Chen and Wang [4]. We analyze the presence and stability of different steady states, as well as their local 
stability by using these modelling frameworks. We also perform a detailed bifurcation analysis to demonstrate the relevant dynamical behaviors of both systems and highlight the interplay of prey refuge, herd behaviour mechanism and predator handling time in shaping community dynamics.

\section{A prey-predator system involving prey refuge}

This section investigates a dynamic system of prey and predator with a response function of Holling type II that incorporates a refuge capacity in prey species. The system proposed by Kar [2] is defined as follows:

$$
\begin{aligned}
\frac{d x}{d t} & =h x\left(1-\frac{x}{k}\right)-\frac{b(1-m) x y}{1+a(1-m) x} \\
\frac{d y}{d t} & =-d y+\frac{c b(1-m) x y}{1+a(1-m) x}
\end{aligned}
$$

where $x$ and $y$ represent the prey and predator species, respectively. All parameters $h, k, a, b, c, d$ are assumed as a positive constant and $m \in[0,1)$ denote a constant proportion of prey that employs refuge. Here $h$ denotes the rate at which prey grows naturally; $k$ is the prey's carrying capacity; $a$ is the amount of time a predator spends handling a single prey; $b$ is the rate at which predators attack and capture the preys; $c$ is the conversion rate at which each prey is converted into a predator's new-born and $d$ is predator death rate. There are at most three steady states in the system (1):

(i) The trivial steady state $E_{0}(0,0)$. This steady state describes the extinction of both prey and predator.

(ii) A boundary steady state $E_{1}(k, 0)$. This steady state indicates the survival of prey and the absence of predator.

(iii) The interior steady state $E_{2}\left(x^{*}, y^{*}\right)$ where $x^{*}=\frac{d}{(c b-a d)(1-m)}$ and $y^{*}=\frac{(a d k m-b c k m-a d k+b c k-d) c h}{k(m-1)(a d-b c)(a d m-b c m-a d+b c)}$. This steady state demonstrates coexistence of both prey and predator.

Next, the Jacobian matrix are employed to conduct stability analysis of these three steady states:

$$
J(x, y)=\left[\begin{array}{cc}
h-\frac{2 h x}{k}-\frac{b(1-m) y}{[1+a(1-m) x]^{2}} & \frac{-b(1-m) x}{1+a(1-m) x} \\
\frac{c b(1-m) y}{[1+a(1-m) x]^{2}} & -d+\frac{c b(1-m) x}{1+a(1-m) x}
\end{array}\right]
$$

Then, the stability of each steady state is investigated. For $E_{0}(0,0)$, the Jacobian of the system is presented by

$$
J(0,0)=\left[\begin{array}{cc}
h & 0 \\
0 & -d
\end{array}\right]
$$

Hence, the eigenvalues of this system are $\lambda_{1}=h, \lambda_{2}=-d$. Since the sign of both eigenvalues is positive and negative, it is concluded that $E_{0}(0,0)$ is an unstable saddle node.

The Jacobian matrix for $E_{1}(k, 0)$ is denoted by 


$$
J(k, 0)=\left[\begin{array}{cc}
-h & \frac{-b(1-m) k x}{h+h k a(1-m)} \\
0 & \frac{-d+c b(1-m) h k}{h+h k a(1-m)}
\end{array}\right]
$$

The eigenvalues of the matrix are $\lambda_{1}=-h, \lambda_{2}=\frac{-d+c b(1-m) h k}{h+h k a(1-m)}$. Since both eigenvalues are negative, it is concluded that $E_{1}(k, 0)$ is locally asymptotically stable when $m>1-\frac{d}{k(c b-a d)}$.

For $E_{2}\left(x^{*}, y^{*}\right)=\left(\frac{d}{(c b-a d)(1-m)}, \frac{(a d k m-b c k m-a d k+b c k-d) c h}{k(m-1)(a d-b c)(a d m-b c m-a d+b c)}\right)$, we compute the Jacobian matrix using the Maple linalg package. And thus, the stability of this steady state is examined in terms of a variety of ecologically significant parameters.

\section{A prey-predator system involving prey refuge and herd behavior in preys}

The prey-predator system is initially presented by Chen and Wang [4]. They assume that preys live in herds, and the term with a square root is introduced in prey density. Thus, the following system is defined as follows:

$$
\begin{aligned}
& \frac{d x}{d t}=h x\left(1-\frac{x}{k}\right)-\frac{b \sqrt{(1-m) x} y}{1+a \sqrt{(1-m) x}} \\
& \frac{d y}{d t}=-d y+\frac{c b \sqrt{(1-m) x} y}{1+a \sqrt{(1-m) x}}
\end{aligned}
$$

where all the parameters are described in the previous section. The steady state in the system (2) are:

(i) The trivial steady state, $P_{0}(0,0)$ describes the extinction of both prey and predator.

(ii) A boundary steady state, $P_{1}(k, 0)$ indicates the survival of prey and the absence of predator.

(iii) The interior steady state, $P_{2}\left(x^{*}, y^{*}\right)$ where $x^{*}=\frac{d^{2}}{(c b-a d)^{2}(1-m)}$ and $y^{*}=\frac{c h d b^{2}\left[k(c b-a d)^{2}(1-m)-d^{2}\right]}{k(c b-a d)^{4}(1-m)^{2}}$. This steady state demonstrates coexistence of both prey and predator.

The given Jacobian matrix determines the stability analysis of the three steady states.

$$
J(x, y)=\left[\begin{array}{ll}
A & B \\
C & D
\end{array}\right]
$$

where 


$$
\begin{aligned}
& A=h-\frac{2 h x}{k}-\frac{b(1-m) y}{2(1+a \sqrt{(1-m) x})}\left[\frac{1}{\sqrt{(1-m) x}}-\frac{a}{(1+a \sqrt{(1-m) x})}\right] \\
& B=\frac{-b \sqrt{(1-m) x}}{1+a \sqrt{(1-m) x}} \\
& C=\frac{c b(1-m) y}{2(1+a \sqrt{(1-m) x})}\left[\frac{1}{\sqrt{(1-m) x}}-\frac{a}{(1+a \sqrt{(1-m) x})}\right] \\
& D=-d+\frac{c b \sqrt{(1-m) x}}{1+a \sqrt{(1-m) x}}
\end{aligned}
$$

Since the system (2) is not linearizable at point $(0,0)$, the stability at $P_{0}(0,0)$ cannot be determined. Therefore, the Jacobian matrix becomes indeterminate since the square root term are incorporated in the system. Meanwhile, for $P_{1}(k, 0)$ and $P_{2}\left(x^{*}, y^{*}\right)$, the eigenvalues cannot be computed analytically due to the Jacobian matrix's complexity and the number of parameters. Due to this, the stability analysis is described in further detail in [4].

\section{Results and discussion}

\section{Dynamical behaviors of prey refuge and herding mechanism}

This section illustrates the numerical simulation of both systems. We performed some simulations using MATLAB and XPPAUT package to investigate systems dynamics as the ecologically significant parameter $m$ (prey refuge) is varied. Then, we will demonstrate some phase portraits and time series analysis for different parameter values $m$. Table 1 shows the parameter values used for both ecological systems (1) and (2).

Table 1. Parameter values used in system (1) and (2).

\begin{tabular}{ccc}
\hline Parameter & Description & Value \\
\hline$h$ & Growth rate of prey & 5 \\
$k$ & Carrying capacity of prey & 60 \\
$m$ & Proportion of prey refuge & system (1): $0.85($ varied $)$ \\
$a$ & Handling time & system (2): $0.4($ varied $)$ \\
$b$ & Attack rate & 0.24 \\
$c$ & Conversion rate & 1.2 \\
$d$ & Death rate of predator & 0.4 \\
\hline
\end{tabular}

We discovered numerous forms of behavior by using the bifurcation analysis as the bifurcation parameter $m$ is varied. Figure 1(a) depicts the prey species' steady state, $x$ versus prey refuge, $m$ for system (1). At $m=m_{1 H}$, the Hopf bifurcation was observed. As $m<m_{1 H}$, the two-species steady states are unstable and stable limit cycle (green dots) is born at $m=m_{1 H}$. However, if as the parameter $m$ increases, as $m_{1 H}>m>m_{1 T}$, the two-species steady state becomes stable. This scenario will allow species to coexist and, as a result, increase species biodiversity. At $m=m_{1 T}$, a transcritical bifurcation occurs. For $m>m_{1 T}$ , the steady state of a single species is stable. This phenomenon indicates that when prey migrates from their habitat patches to the refuge, they are no longer vulnerable to predation. Consequently, the prey population reached the carrying capacity (maximum population size that a particular area can sustainably support). As a result, predators will be unable to survive.

Similar observations are realized in Figure 1(b); the prey species' steady state, which employs herd behavior and group defense mechanism, $x$ against prey refuge, $m$. There occur threshold values $m_{2 H}$ and $m_{2 T}$, which equate to Hopf and transcritical bifurcation points, respectively. 
As the prey refuge $m$ decreases $\left(m<m_{2 H}\right)$, stable limit cycles grow in amplitude until they collide with extinction steady states in homoclinic bifurcation (blue dots in Figure 1(b)) and hence, the limits cycles will completely disappear [7]. Theoretically, the homoclinic bifurcation refers to a destruction or disappearance of the limit cycle. In this case, a limit cycle eventually reaches a saddle point and transforms into a homoclinic orbit at the bifurcation [8]. Ecologically, this condition may result in the absence of prey species, which is followed by the absence of predator species.

Compared to system (1), it is clear that the bifurcation threshold values are shifted to the left (see Figure 1(a)). This scenario demonstrates that prey populations live in herds and then employ prey refuges, which is possible to reduce predator density and predation rates. As a result, this condition will facilitate the coexistence of the two species. The stability and bifurcation analysis of systems (1) and (2) also can be summarized and classified into Table 2 and 3, respectively.

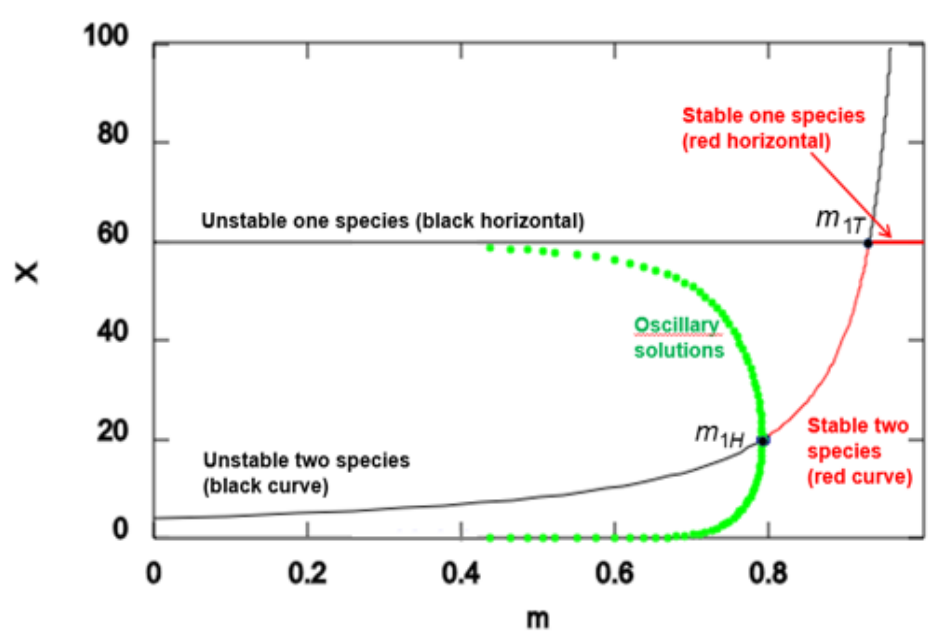

(a) Density of species $x$ of system (1) against $m$

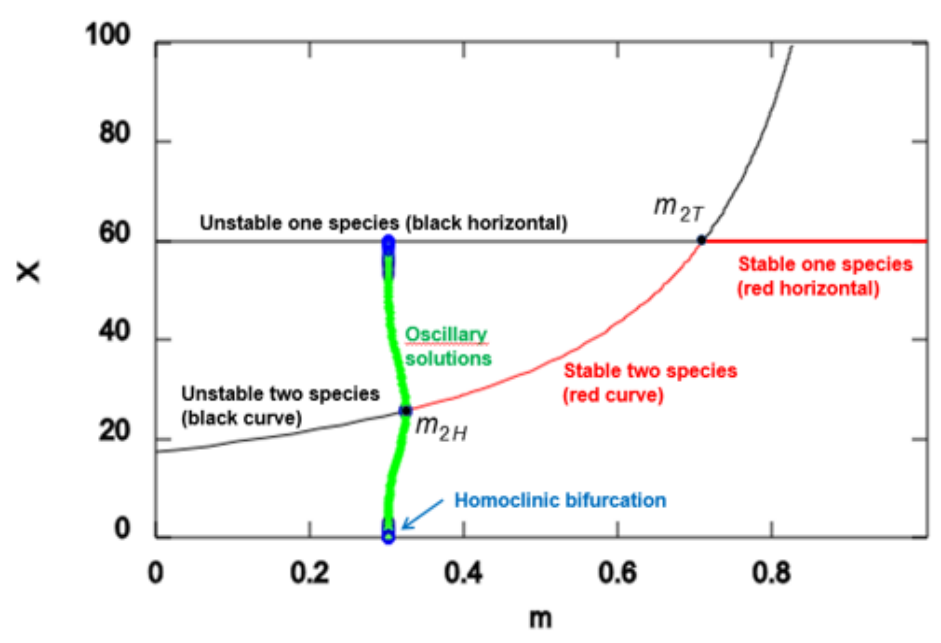

(b) Density of species $x$ of system (2) against $m$

Figure 1. One-parameter bifurcation diagram as species $x$ against $m$ (prey refuge) is varied. The points of $m_{1 T}$ and $m_{2 T}$ are the transcritical bifurcation, while $m_{1 H}$ and $m_{2 H}$ are the Hopf bifurcation points. The red and black curves represent the stable and unstable steady states, respectively. The oscillatory solutions are represented by the green dots (i.e., stable limit cycle). The blue dots in (b) are the homoclinic bifurcation.

Table 2. The numerical result of system (1).

\begin{tabular}{|c|c|c|c|c|}
\hline $\begin{array}{l}\text { Parameter } \\
\text { values }\end{array}$ & Steady states & Eigenvalues & Characteristics & Figure \\
\hline$m=0.6$ & $E_{2}(10.42,17.22)$ & $\lambda=0.599 \pm 1.3067 i$ & Unstable spiral steady state (stable limit cycle exists) & 2 \\
\hline$m=0.7917$ & \multicolumn{3}{|c|}{ Hopf bifurcation point $\left(m_{1 H}\right)$} & $1(a)$ \\
\hline$m=0.85$ & $E_{2}(27.78,29.84)$ & $\lambda=-0.4861 \pm 1.0518 i$ & Asymptotically stable spiral steady state & 3 \\
\hline$m=0.9306$ & \multicolumn{3}{|c|}{ Transcritical bifurcation point $\left(m_{1 T}\right)$} & $1(\mathrm{a})$ \\
\hline$m=0.95$ & $E_{1}(60,0)$ & $\begin{array}{l}\lambda_{1}=-0.1628 \\
\lambda_{2}=-5\end{array}$ & Local asymptotically stable steady state & 4 \\
\hline
\end{tabular}


Table 3. The numerical result of system (2).

\begin{tabular}{|c|c|c|c|c|}
\hline $\begin{array}{l}\text { Parameter } \\
\text { values }\end{array}$ & Steady states & Eigenvalues & Characteristics & Figure \\
\hline$m=0.31$ & $P_{2}(25.16,29.22)$ & $\lambda=0.0403 \pm 0.851 i$ & Unstable spiral steady state (stable limit cycle exists) & 5 \\
\hline$m=0.3248$ & \multicolumn{3}{|c|}{ Hopf bifurcation point $\left(m_{2 H}\right)$} & 1 (b) \\
\hline$m=0.4$ & $P_{2}(28.94,29.96)$ & $\lambda=-0.2349 \pm 0.7694 i$ & Asymptotically stable spiral steady state & 6 \\
\hline$m=0.7106$ & \multicolumn{3}{|c|}{ Transcritical bifurcation point $\left(m_{2 T}\right)$} & $1(\mathrm{~b})$ \\
\hline$m=0.8$ & $P_{1}(60,0)$ & $\begin{array}{l}\lambda_{1}=-0.092 \\
\lambda_{2}=-5\end{array}$ & Local asymptotically stable steady state & 7 \\
\hline
\end{tabular}

Each vertical slice of the bifurcation diagram (see Figure 1) for a specific parameter value $m$ (see Tables 1 and 2) represents a phase portrait and time series diagrams of the dynamical system (1) and (2) in Figures 2 - 7.

Using the phase portrait and time series analysis, we observed that various outcomes of species dynamics emerge when the prey refuge, $m$ are varied. Figure 2 (a) depicts the phase portrait of both species in the presence of a modest population density of prey refuge (e.g., $m=0.6$ ) in the system (1). There is an oscillatory solution (see Figure $2(b)$ ), in which the population densities of both species begin to fluctuate over time. In this situation, the steady state of the coexistence species is unstable, and trajectories from any positive initial conditions converge to a stable limit cycle (see Figure 2 (a)).

As seen in Figures 3(a) and 3(b), the coexisting species steady state stabilizes when the rate of prey refuge increases (e.g., $m=0.85$ ). This scenario explained the allowing for more better dispersion in terms of prey refuge appears to have a rescuing impact on prey and encourages the coexistence of various species. Nevertheless, the high prey refuge densities (e.g., $m=0.95$ ) may be harmful to coexistence since only prey species can survive until they reach their carrying capacity (see Figure 4 (a) and (b)).

Figures 5 - 7 show the phase portrait and time series plots of system (2) as the prey employ both refuge and herding mechanisms. In this case, we discover relatively similar dynamics to those observed in the system (1). However, numerous possible outcomes of species interactions exist, including oscillatory patterns (see Figure $5(\mathrm{a})$ and (b)), coexistence of both species (see Figure $6(\mathrm{a})$ and (b)), and species absence (see Figure 7 (a) and (b)) for $m=0.31, m=0.4$, and $m=0.8$, respectively. Conversely, when refuge intensity in prey species is low (e.g., $m=0.2$ ), this eventuality results in the termination of stable limit cycles.

Our findings reveal that both systems (1) and (2) can exhibit various dynamical behaviors. As prey refuge $m$ varies, several stable attractors may emerge, including limit cycles, single-species steady states, and the coexistence of two-species steady states. The following sections illustrate how the combined effects of prey refuge, herd behaviour mechanism and predator handling time might impact the composition of species communities. To address this concern, two-parameter bifurcation analysis of the systems (1) and (2) is performed. 


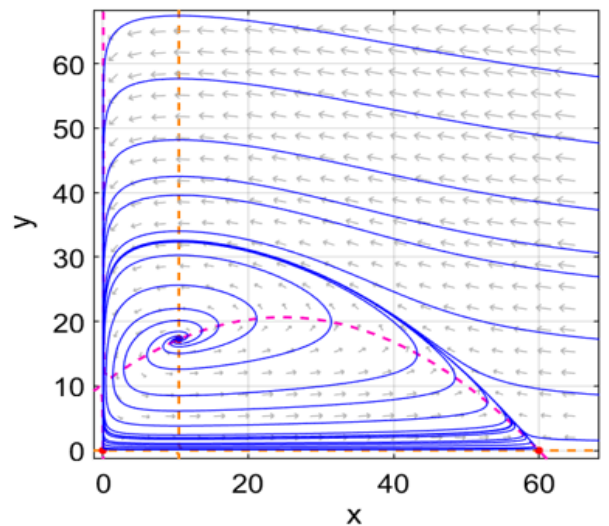

(a)

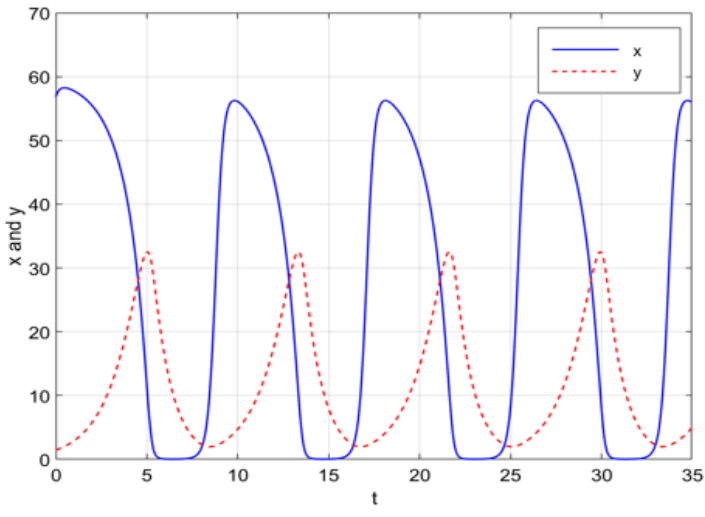

(b)

Figure 2. The numerical illustrations for system (1) with $m=0.6$ and initial condition $\left(x_{0}, y_{0}\right)=(58,2)$. (a) The phase plane, prey $(x)$ vs. predator $(y)$, and (b) the time series plot $(x, y$ vs. $t)$.

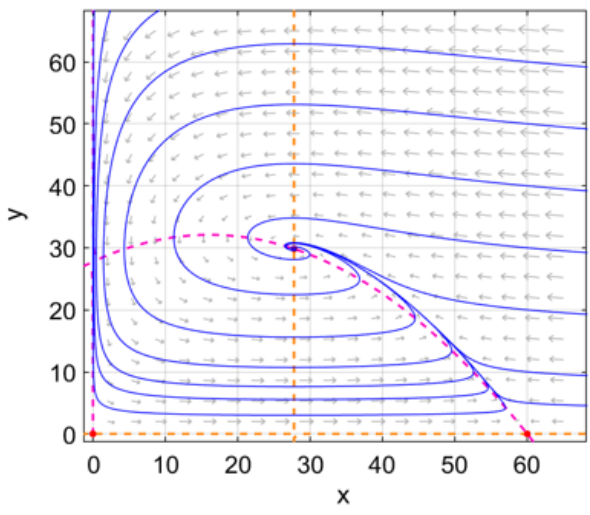

(a)

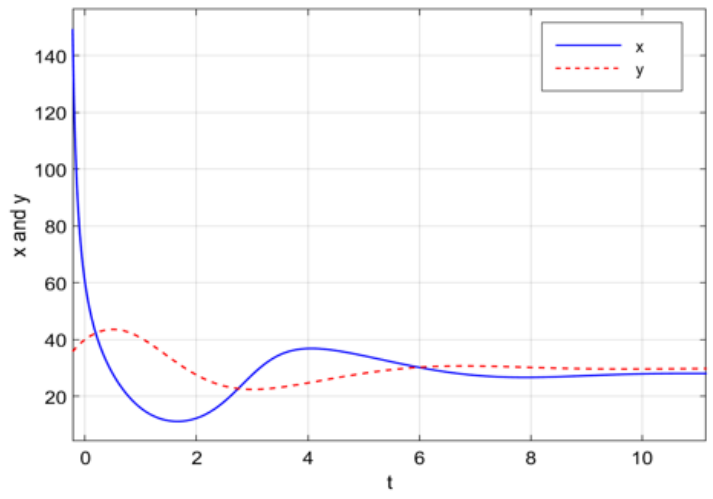

(b)

Figure 3. The numerical illustrations for system (1) with $m=0.85$ and initial condition $\left(x_{0}, y_{0}\right)=(150,38)$. (a) The phase plane, prey $(x)$ vs. predator $(y)$, and (b) the time series plot $(x, y$ vs. $t)$.

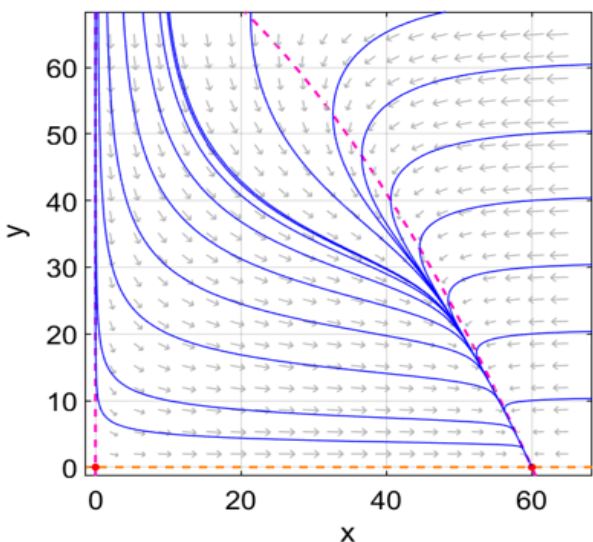

(a)

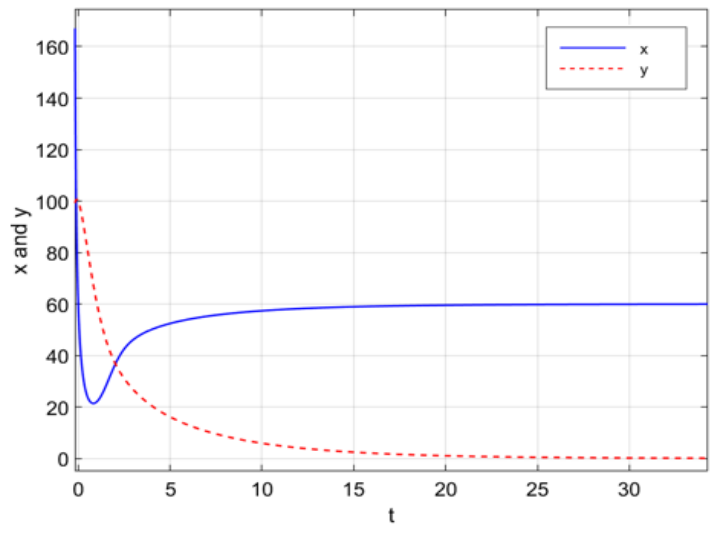

(b)

Figure 4. The numerical illustrations for system (1) with $m=0.95$ and initial condition $\left(x_{0}, y_{0}\right)=(165,100) \cdot(a)$ The phase plane, prey $(x)$ vs. predator $(y)$, and (b) the time series plot $(x, y$ vs. $t)$. 


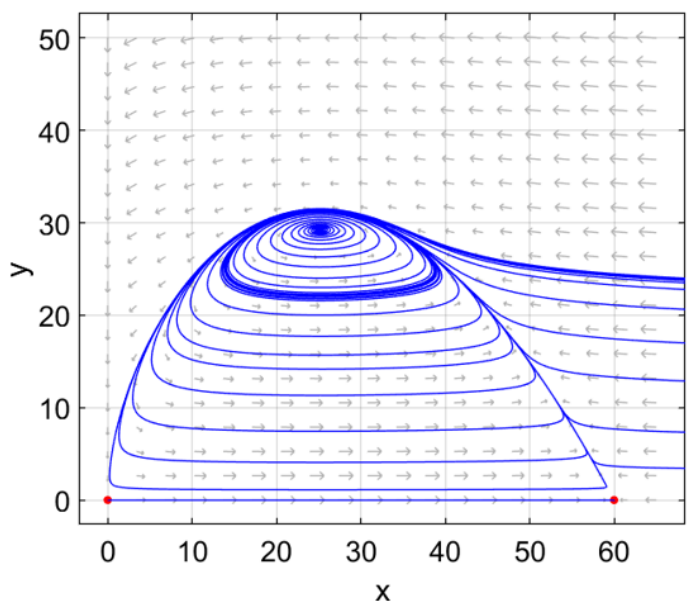

(a)

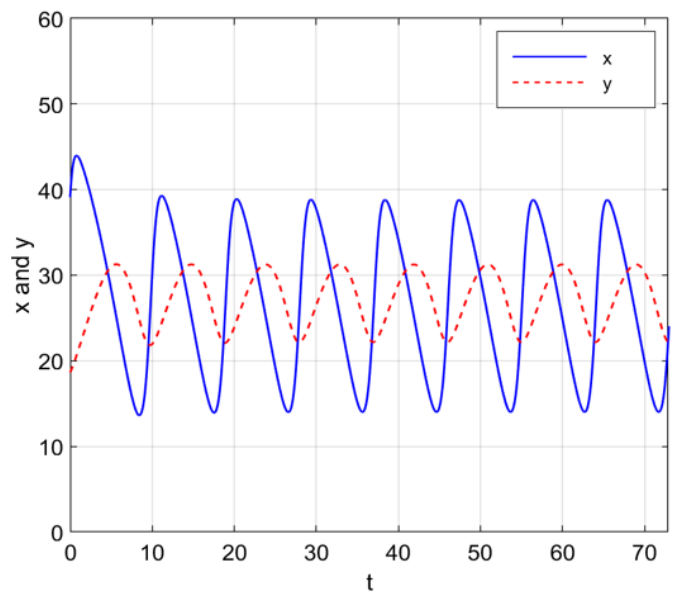

(b)

Figure 5. The numerical illustrations for system (2) with $m=0.31$ and initial condition $\left(x_{0}, y_{0}\right)=(39,19)$. (a) The phase plane, prey $(x)$ vs. predator $(y)$, and (b) the time series plot $(x, y$ vs. $t)$.

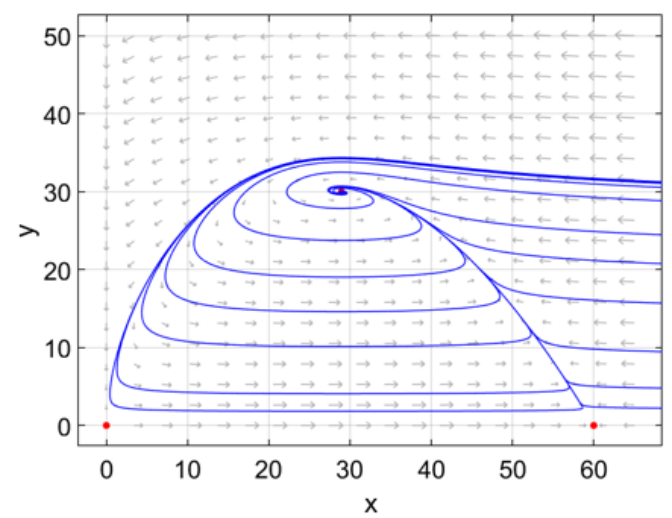

(a)

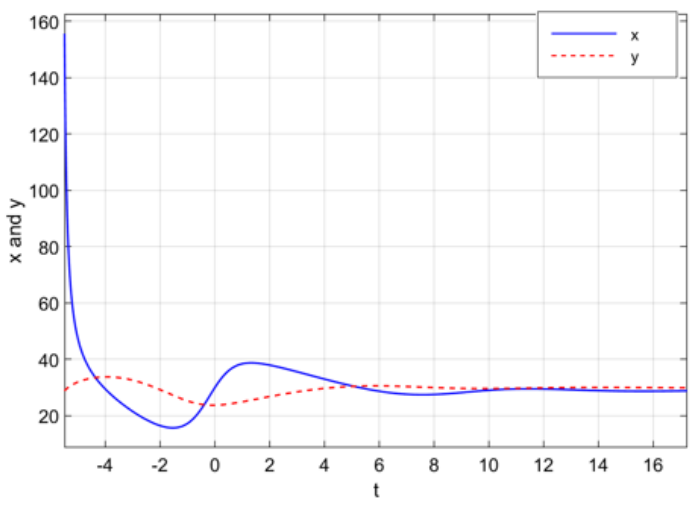

(b)

Figure 6. The numerical illustrations for system (2) with $m=0.4$ and initial condition $\left(x_{0}, y_{0}\right)=(158,25) \cdot(a)$ The phase plane, prey $(x)$ vs. predator $(y)$, and (b) the time series plot $(x, y$ vs. $t)$.

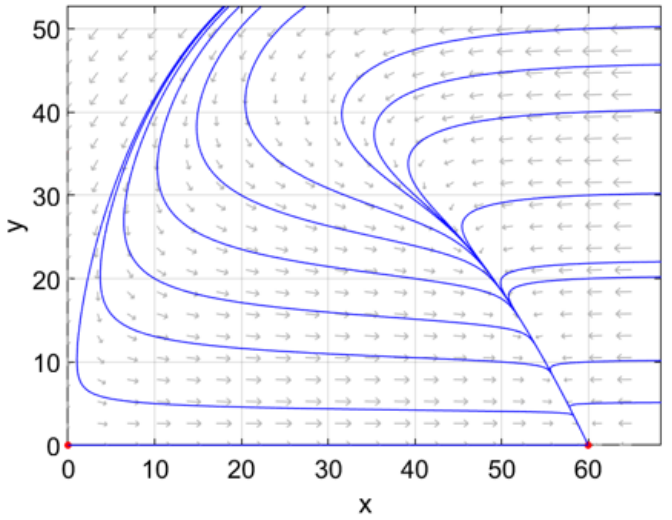

(a)

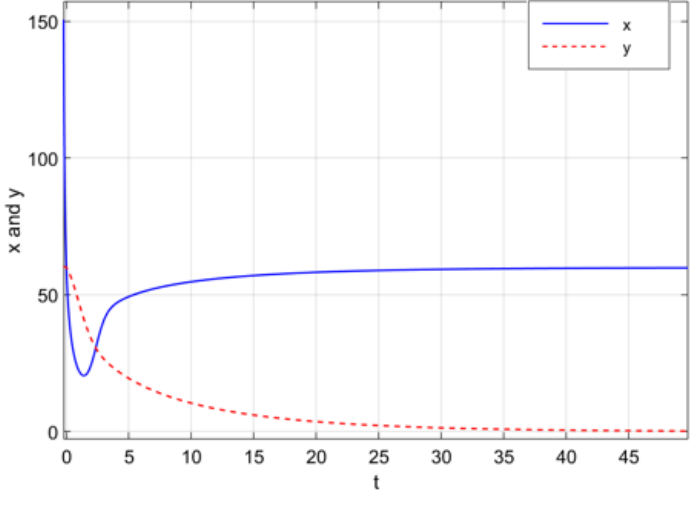

(b)

Figure 7. The numerical illustrations for system (2) with $m=0.8$ and initial condition $\left(x_{0}, y_{0}\right)=(150,55)$. (a) The phase plane, prey $(x)$ vs. predator $(y)$, and (b) the time series plot $(x, y$ vs. $t)$. 
The combined influence of predator handling time and prey refuge

As illustrated in Figure 8, the two-parameter bifurcation diagram is constructed to investigate the combined influence of prey refuge $m$, predator handling time a (the amount of time a predator spends handling one prey) and herd behaviour mechanism. The stability regions are divided into several parts: (i) the blue region denotes the presence of stable limit cycles.; (ii) the green region represents twospecies steady states in which both species coexist.; (iii) the red region represents the steady-state of a single species with the exclusion of predator species; (iv) yellow region (see Figure 8(b)) corresponds to the absence of stable limit cycles.

Figure 8 shows the two-parameter bifurcation diagram for both systems (1) and (2). The red region represents situations in which prey disperses rapidly, and predators have a high handling time rate. This situation endangers the survival of both species. It seems there is a single species steady state in this area, which results in the existence of prey while predator species are wiped out. As we pass through the transcritical bifurcation curve and enter the green region, the steady state for two-species coexistence stabilizes, allowing both species to coexist in the ecosystem sustainably. Shifting into the blue region as we traverse the Hopf bifurcation curve, the oscillatory behaviors emerge, indicating the presence of a stable limit cycle. But then, the stable limit cycle eventually terminates as we reach the homoclinic bifurcation curve in Figure 8(b).
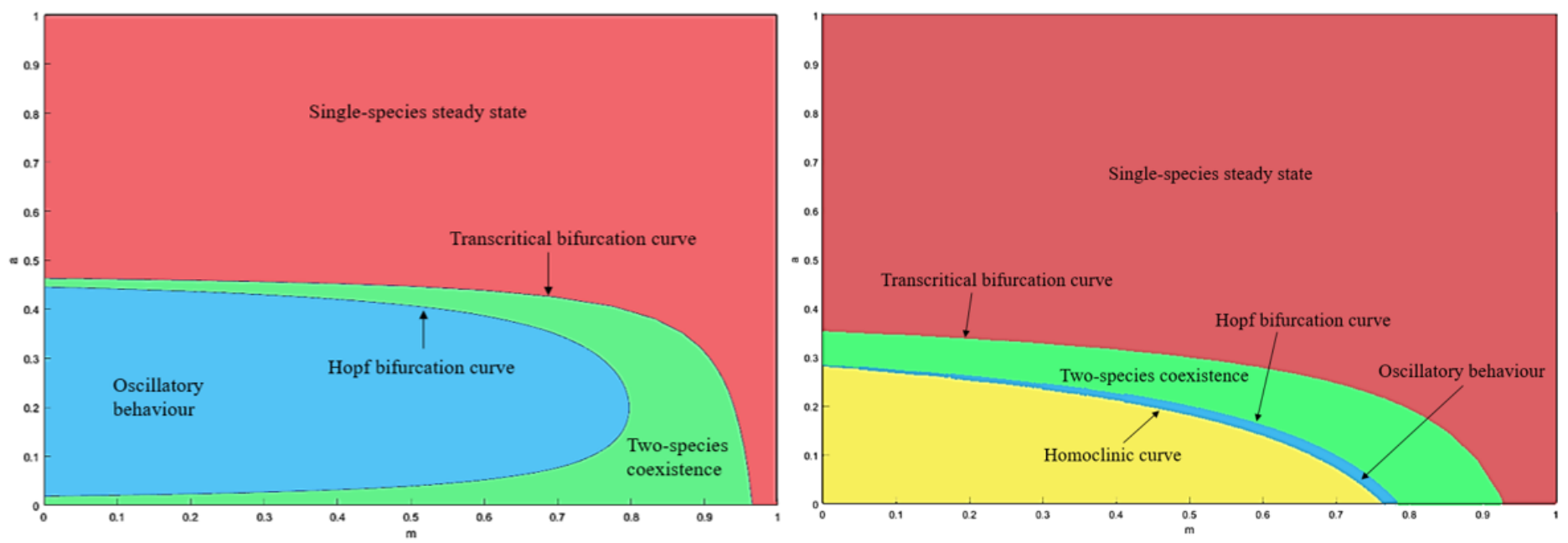

Figure 8. Diagrams of a two-parameter bifurcation analysis for a (predator handling time) against $m$ (prey refuge). (a) System (1) employ prey refuge. (b) System (2) incorporates prey refuge and herd mechanism.

In comparison, if the predator handling time is kept to a minimum, e.g., $a=0.001$, this situation enhances a broader range of two-species coexistence green areas in Figure 8(a). However, when prey employs the herding mechanism, the green region of coexistence between two species shrinks, as illustrated in Figure 8(b). Furthermore, the red region in Figure 8(b) is significantly larger than the equivalent region in Figure 8(a). This situation indicates that predator handling time in system (2) is longer than in system (1) since the prey employed the herd mechanisms. Ecologically, increasing predator handling times may be harmful to species diversity [9]. More possibilities involving species exclusion are feasible across an ecological variation of prey refuge [10]. The stable limit cycles with an unstable two-species steady-state can lead to instability in the predator-prey ecosystem, possibly resulting in local eradication or even a species extinction [11]. However, the stable limit cycles in the system (2) become smaller compared to the system (1). This scenario demonstrates that employing herd behaviour mechanisms in prey can reduce the likelihood of a species being extirpated. 


\section{Conclusions}

The dynamics of two ecological prey and predator systems are considered to understand better the combined effects of prey refuge and herd behaviour mechanisms in determining species population dynamics. Correspondingly, system (1) employs prey refuge, while system (2) incorporates prey refuge and herd behavior mechanisms. The mathematical softwares MATLAB and XPPAUT are carried out to analyze each bifurcation parameter's stability and bifurcation results.

By using one-parameter bifurcation analysis, it is revealed that both systems (1) and (2) undergo transcritical and Hopf bifurcation. However, since system (2) employs both prey refuge and herd mechanism, a Homoclinic bifurcation occurs. The bifurcation parameter $m$ is then varied, discussed, and classified into three parts: low, moderate, and high prey refuge intensities. We discovered qualitatively similar dynamics on both systems (1) and (2) based on the dynamical analysis. The low intensity of prey refuges will eventually lead to the exclusion of both species. Both systems exhibit twospecies coexistence and, consequently, enhance species biodiversity when prey refuges are moderately intense. Finally, the high intensity of prey refuges would eventually allow prey to reach its carrying capacity, resulting in the exclusion of predators.

For a two-parameter bifurcation analysis, the combined effects of prey refuge and predator handling time are investigated. It can be concluded that the single-species stable region in the system (2) is more extensive than in the system (1). Ecologically, when prey employs both refuge and herd strategies, the predator would spend much more time handling the prey to attack, kill, and consume it. In fact, incorporating both prey refuge and herd mechanisms into the prey population aids in the prey's natural survival. This condition, however, could be threatening to species diversity. To summarize, future research should emphasize prey refuges in combination with herd behaviour mechanisms in both prey and predator populations.

\section{Conflicts of interest}

The author(s) declare(s) that there is no conflict of interest regarding the publication of this paper.

\section{Funding statement}

This research is supported by the Ministry of Higher Education Malaysia for Fundamental Research Grant Scheme with Project Code: FRGS/1/2018/STG06/USM/02/4.

\section{Acknowledgments}

The authors would like to express their gratitude to the Ministry of Higher Education Malaysia for the FRGS grant, as well as the editor and reviewers for their insightful comments and recommendations, which significantly improved the presentation of this manuscript.

\section{References}

[1] E. Gonzalez-Olivares and R. Ramos-Jiliberto, "Dynamic consequences of prey refuges in a simple model system: more prey, fewer predators and enhanced stability," Ecological Modelling, vol. 166, no. 1-2, pp. 135146, 2003.

[2] T. K. Kar, "Stability analysis of a prey-predator model incorporating a prey refuge," Communications in Nonlinear Science and Numerical Simulation, vol. 10, no. 6, pp. 681-691, 2005.

[3] M. H. Mohd, N. F. A. A. Halim and F. N. Zaidun, "Combined effects of prey refuge and death rate of predator on the prey-predator population dynamics," AIP Conference Proceedings, vol. 2184, no. 1, p. 060028, 2019.

[4] L. Chen and Y. Wang, "Dynamical analysis on prey refuge in a predator-prey model with square root functional response," J. Math. Computer Sci., vol. 18, no. 2, pp. 154-162, 2018.

[5] L. Chen and F. Chen, "Global analysis of a harvested predator-prey model incorporating a constant prey refuge," International Journal of Biomathematics, vol. 3, no. 2, pp. 205-223, 2010. 
[6] T. K. Kar, "Modelling and analysis of a harvested prey-predator system incorporating a prey refuge," Journal of Computational and Applied Mathematics, vol. 185, no. 1, pp. 19-33, 2006.

[7] Z. I. A. Manaf and M. H. Mohd, "Bifurcation analysis of the prey-predator models incorporating herd behaviours," IOP Conference Series: Earth and Environmental Science, vol. 380, no. 1, p. 012009, 2019.

[8] S. H. Strogatz, "Nonlinear dynamics and chaos with student solutions manual: With applications to physics, biology, chemistry, and engineering", CRC press, 2018.

[9] S. J. Schreiber and M. Vejdani, "Handling time promotes the coevolution of aggregation in predator-prey systems," Proceedings of the Royal Society B: Biological Sciences, vol. 273, no. 1583, pp. 185-191, 2006.

[10] M. H. Mohd and M. S. M Noorani, "Local dispersal, trophic interactions and handling times mediate contrasting effects in prey-predator dynamics," Chaos, Solitons \& Fractals, vol. 142, no. -, p. 110497, 2021.

[11] M. L. Rosenzweig, "Paradox of enrichment: destabilization of exploitation ecosystems in ecological time," Science, vol. 171, no. 3969, pp. 385-387, 1971. 